\title{
Secretory Carcinoma Arising in a Fibroadenoma: A Brief Case Report
}

\author{
Sharon Lim $\cdot$ Min Keun Shim ${ }^{1}$ Eun Yoon $\mathrm{Cho}^{2} \cdot$ Soo Youn $\mathrm{Cho}^{2}$ \\ Department of Pathology, Hanyang University Guri Hospital, Hanyang University College of Medicine, Guri; ' ${ }^{1}$ Foryou Pathology Laboratory, Gwangju; \\ ${ }^{2}$ Department of Pathology and Translational Genomics, Samsung Medical Center, Sungkyunkwan University College of Medicine, Seoul, Korea
}

Secretory carcinoma is a rare subtype of breast cancer, accounting for less than $0.1 \%$ of invasive breast cancers. ${ }^{1}$ It is characterized by distinctive intracellular and extracellular secretory material in solid, microcystic, and tubular growth patterns.

Recently, we experienced this rare tumor arising in a fibroadenoma (FA). Ductal epithelium within FA can undergo carcinomatous change. There have been several case reports of carcinoma arising in FA, usually in forms of lobular or ductal carcinoma in situ. ${ }^{2-4}$ However, there has been no reported case of secretory carcinoma arising in a FA.

\section{CASE REPORT}

A 20-year-old woman visited a local hospital with a palpable nodule in the right upper center breast. Her family history and past history were unremarkable. Also she denied the possibility of pregnancy or oral contraceptive use. She underwent excision of the mass under the clinical impression that it was benign. Seven months later, she visited Samsung Medical Center for a second opinion. Mammography and ultrasonography revealed no residual mass or abnormality.

At low power view, the tumor, measuring $0.7 \times 0.6 \mathrm{~cm}$, was multinodular with intervening fibrous septa. There were multifocal, less cellular, myxoid areas at the periphery (Fig. 1A). Tumor cells showed solid sheets or cribriform arrangement, and the tumor nests showed microcystic structures containing pale bluish secretory

\section{Corresponding Author}

Soo Youn Cho, MD, PhD

Department of Pathology and Translational Genomics, Samsung Medical Center Sungkyunkwan University School of Medicine, 81 Irwon-ro, Gangnam-gu, Seoul 06351, Korea

Tel: +82-2-3410-2817, Fax: +82-2-3410-0025, E-mail: sooyoun.cho@samsung.com

Received: May 8, 2017 Revised: July 31, 2017

Accepted: August 1, 2017 material (Fig. 1B). The tumor cells were large and polygonal, displaying abundant vacuolated cytoplasm with intracellular secretory material. The secretory material was periodic acid Schiffpositive (Fig. 1C). Nuclei were small, showing mild to moderate atypia and small nucleoli. Bloom-Richardson histologic grade was grade II. There was no lymphovascular invasion. The tumor cells were diffusely positive for S-100 protein (1:5,000, polyclonal, Dako, Capinteria, CA, USA) (Fig. 1D). At the periphery of the mass, bland tubular ducts were mixed with nests of tumor cells within myxoid stroma (Fig. 1E, F). The tumor cell nests within myxoid stroma showed diffuse strong positivity for S-100 protein, while the bland tubular components were negative (Fig. 1G). Calponin (1:200, clone CALP, Dako) and p63 (1:200, clone 4A4, Biocare Medical, Concord, CA, USA) staining confirmed the presence of myoepithelial cells in the bland tubular components (Fig. 1H). A few tumor cells expressed p63. This lesion corresponded to the pericanalicular pattern of FA. The invasive portion was confined within the FA with no extension to adjacent parenchyma. A few ducts with secretory type ductal carcinoma in situ were noted in and outside the boundary of FA (Fig. 1I, J). Estrogen receptor (ER; 1:200, clone 6F11, Novocastra Laboratories, Newcastle upon Tyne, UK), progesterone receptor (PR; 1:100, clone 16, Novocastra Laboratories, Newcastle upon Tyne, UK), and human epidermal growth factor receptor 2 (HER-2; clone 4B5, Ventana Medical Systems, Inc., Tucson, AZ, USA), epidermal growth factor receptor (1:100, clone EGFR.25, Novocastra Laboratories) were all negative in tumor cells, while cytokeratin (CK) 5/6 (1:100, clone D5/16B4, Dako) was expressed in 5\% of the tumor cells. Ki-67 (1:200, clone MIB-1, Dako) labeling index was about 5\%. ETV6 rearrangement was confirmed by ETV6 break-apart fluorescence in situ hybridization (Abbott Molecular, Chicago, IL, USA). We diagnosed this tumor as secretory carcinoma arising in a FA. Resection margin assessment was not feasible 

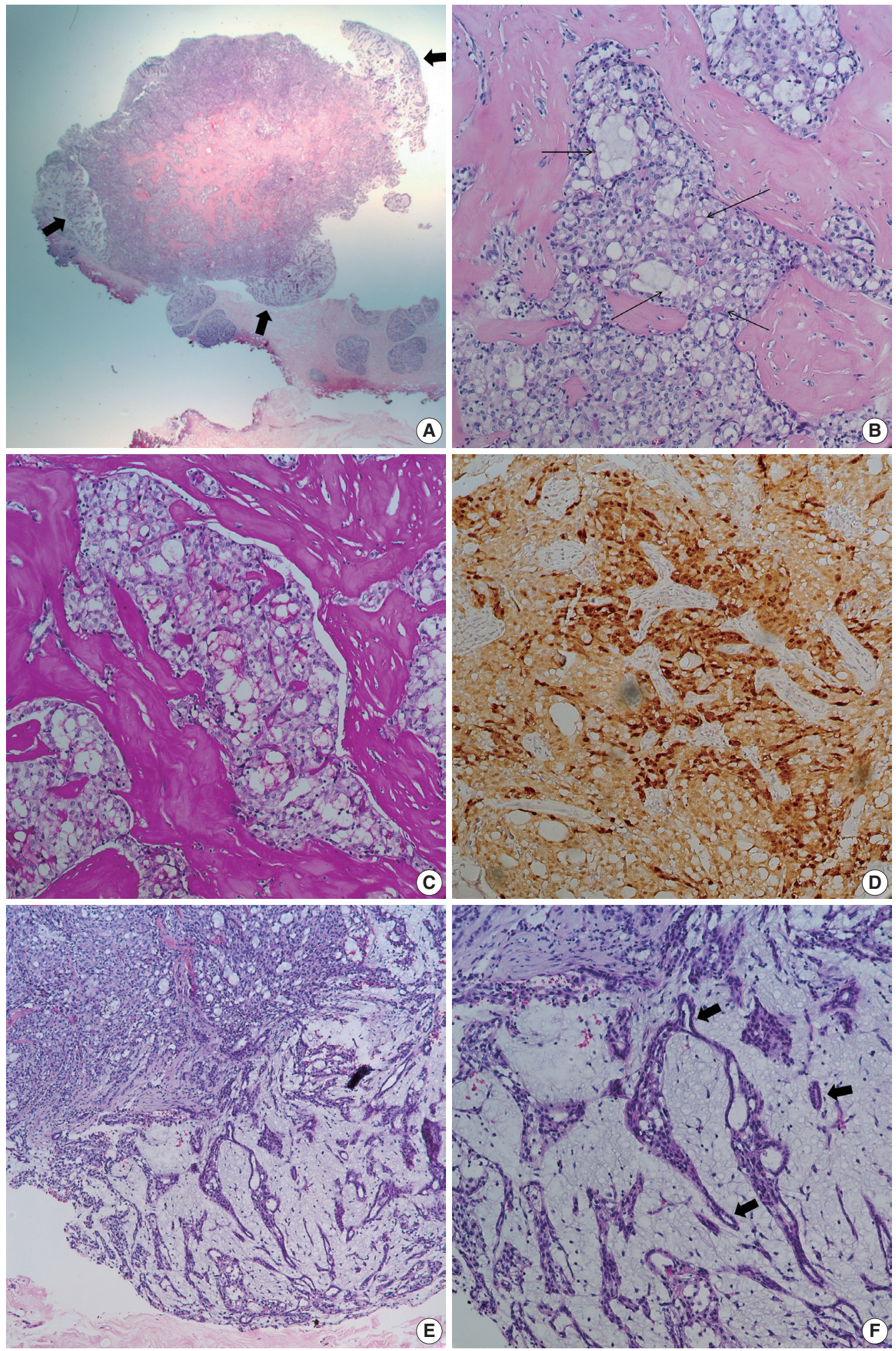

Fig. 1. Microscopic findings. (A) Low power field image shows fibrous septa within the tumor and peripheral hypocellular area (thick arrows). (B) Tumor shows solid or microcystic growth patterns within dense fibrous stroma. Cribriform tumor nests contain extracellular secretory material (thin arrows). Tumor cells have abundant, vacuolated cytoplasm. (C) Periodic acid Schiff staining highlights intra-and extracellular secretory material. (D) Tumor cells are diffusely positive for S-100 protein. (E) At the periphery of cellular tumor (left upper), hypocellular myxoid area is present (right lower). (F) Peripheral myxoid area shows mixture of bland tubules (arrows) and irregular tumor nests.

(Continued to the next page) 

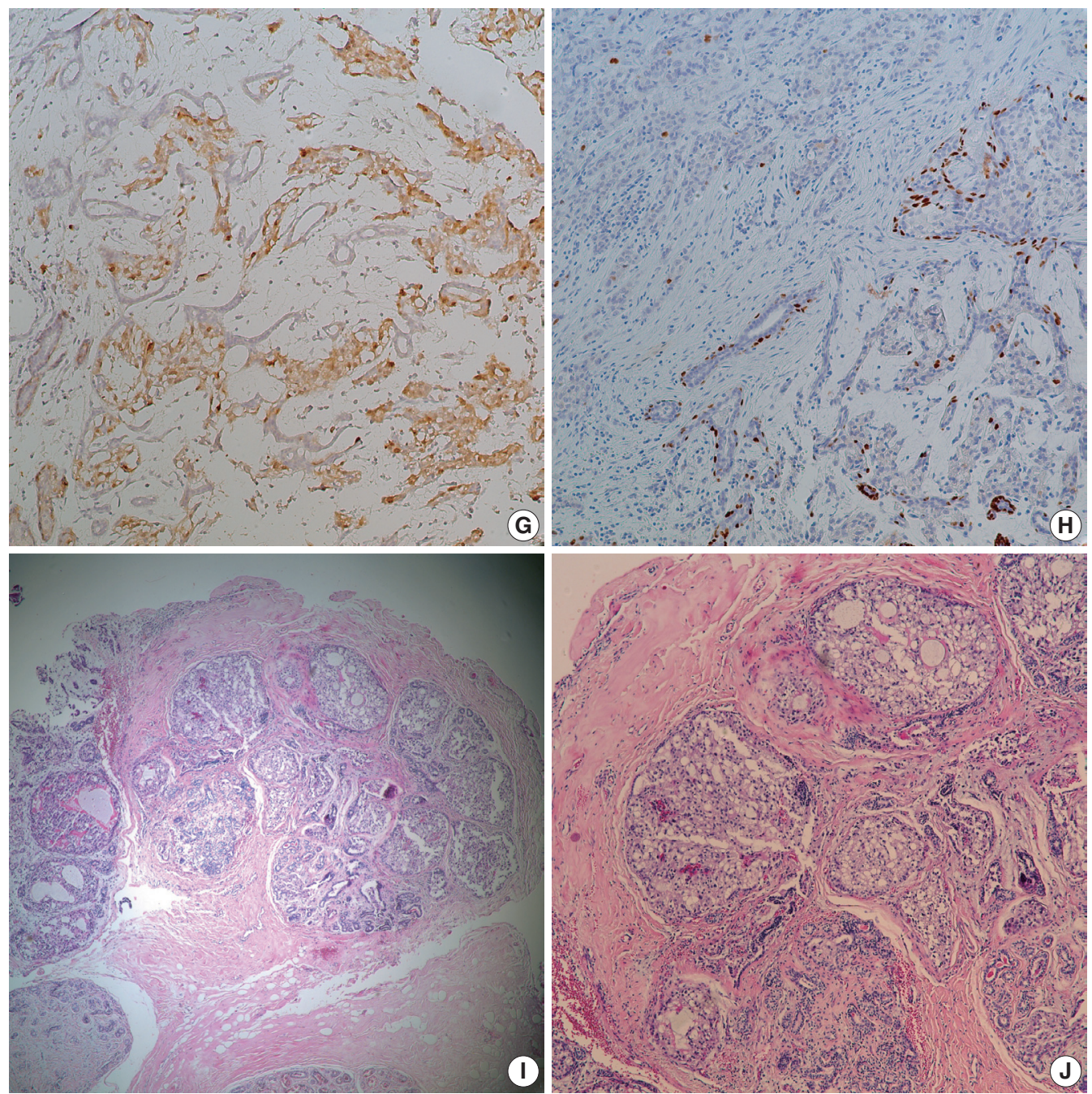

Fig. 1. (Continued from the previous page) $(G)$ Tumor cells are positive for S-100 protein, while bland tubules are negative. (H) p63 staining highlights the presence of myoepithelial cells in the myxoid area. A few tumor cells express p63. (I) Ductal carcinoma in situ (DCIS) is noted in (left side) and outside the boundary of fibroadenoma. (J) DCIS also shows intracellular and extracellular secretory material.

due to tissue fragmentation. No further treatment was performed. The patient is well with no evidence of recurrence at 21 months after excision.

This study was approved by the Institutional Review Board of Samsung Medical Center, and informed consent was waived (2017-09-078).

\section{DISCUSSION}

Secretory carcinoma was initially described in 1966 as "juvenile breast carcinoma" by McDivitt and Stewart. ${ }^{5}$ Cases occurring in a wider age range were subsequently reported. Thus, the term "juvenile" was abandoned and the new title of "secretory carcinoma" was proposed by Tavassoli and Norris. ${ }^{6}$ Secretory carcinoma exhibits typical microscopic features of solid, microcystic, and glandular growth patterns with intra- and extra-cellular secretory material. Immunophenotypically, secretory carcinoma usually belongs to the triple-negative, basal-like type, which is negative for ER, PR, and HER-2 with expression of basal (CK5/6 and CK14) markers. ${ }^{7}$ However, a recent large scale study from the National Cancer Data Base has reported that ER and PR are expressed in a considerable number of secretory carcinomas (64\% and 44\%, respectively). ${ }^{8} \mathrm{~A}$ balanced translocation, $\mathrm{t}(12 ; 15)$, that causes an ETV6NTRK3 fusion, also characterizes secretory carcinoma. ${ }^{9}$

Contrary to other basal-like type breast carcinomas, secretory carcinoma is a slowly growing, indolent disease with an estimated disease-specific survival of more than $90 \%{ }^{1,10}$ It has been shown that even patients with lymph node metastasis have excellent 
prognosis. ${ }^{10}$ Distant metastasis is rare, but it is associated with large tumor size and multiple lymph node involvement. ${ }^{10}$

Breast cancer arising in a FA is an unusual presentation. Most reported cases have been lobular and ductal carcinoma in situ. ${ }^{2}$ Some of the carcinomas were confined within the FA, while others showed extension beyond the FA. ${ }^{2}$ In the case of carcinoma in situ arising in a FA, a more favorable prognosis has been suggested. ${ }^{2}$ However, it is unknown whether the prognosis of carcinoma arising in a FA would differ from that of usual carcinoma because of the limited number of cases. It can be stated that in the case of carcinoma arising in $\mathrm{FA}$, the FA attributes to a more favorable prognosis by enabling an earlier detection of the breast cancer due to mass effect. ${ }^{2-4}$

We described an unusual case of secretory carcinoma arising within a FA. To our knowledge, this is the first report of secretory carcinoma arising in a FA. Recognizing the FA component might be important for patient management.

\section{Conflicts of Interest}

No potential conflict of interest relevant to this article was reported.

\section{REFERENCES}

1. Horowitz DP, Sharma CS, Connolly E, Gidea-Addeo D, Deutsch I. Secretory carcinoma of the breast: results from the survival, epide- miology and end results database. Breast 2012; 21: 350-3.

2. Diaz NM, Palmer JO, McDivitt RW. Carcinoma arising within fibroadenomas of the breast: a clinicopathologic study of 105 patients. Am J Clin Pathol 1991; 95: 614-22.

3. Wu YT, Chen ST, Chen CJ, et al. Breast cancer arising within fibroadenoma: collective analysis of case reports in the literature and hints on treatment policy. World J Surg Oncol 2014; 12: 335.

4. Chintamani, Khandelwal R, Tandon M, et al. Carcinoma developing in a fibroadenoma in a woman with a family history of breast cancer: a case report and review of literature. Cases J 2009; 2: 9348.

5. McDivitt RW, Stewart FW. Breast carcinoma in children. JAMA 1966; 195: 388-90.

6. Tavassoli FA, Norris HJ. Secretory carcinoma of the breast. Cancer 1980; 45: 2404-13.

7. Laé M, Fréneaux P, Sastre-Garau X, Chouchane O, Sigal-Zafrani B, Vincent-Salomon A. Secretory breast carcinomas with ETV6NTRK3 fusion gene belong to the basal-like carcinoma spectrum. Mod Pathol 2009; 22: 291-8

8. Jacob JD, Hodge C, Franko J, Pezzi CM, Goldman CD, Klimberg VS. Rare breast cancer: 246 invasive secretory carcinomas from the National Cancer Data Base. J Surg Oncol 2016; 113: 721-5.

9. Tognon C, Knezevich SR, Huntsman D, et al. Expression of the ETV6-NTRK3 gene fusion as a primary event in human secretory breast carcinoma. Cancer Cell 2002; 2: 367-76.

10. Vasudev P, Onuma K. Secretory breast carcinoma: unique, triplenegative carcinoma with a favorable prognosis and characteristic molecular expression. Arch Pathol Lab Med 2011; 135: 1606-10. 